\title{
Impact of Different Training Mode on Adaptive Equalization Techniques for MIMO-OFDM System
}

\author{
Bashar A. Mohammed \\ Department of Electrical Engineering, \\ College of Engineering, \\ University of Mosul, Mosul, Iraq
}

\author{
Siddeeq Y. Ameen \\ Dean of Research and Graduate Studies \\ Applied Science University \\ East Al-Ekir, Bahrain
}

\begin{abstract}
The paper investigates the performance enhancement of MIMO-OFDM system by using LMS, VSSLMS, SignLMS and RLS adaptive equalizers. Precisely the paper compares between two methods of training mode in equalizers that are used with MIMO-OFDM system, the Full Frame (FF) method that uses one frame from sets of frames as a desired signal and the Part Frame (PF) method uses part of the frame as a desired signal. The investigation aims to determine which method of training is best among the adopted equalizers in terms of tolerance to AWGN, adjustment speed and complexity. This has been achieved via computer simulation of the four equalization techniques mentioned earlier under the two forms of training modes. The results of the investigation show that the FF mode of training is preferable with LMS, VSSLMS and SignLMS and can be resumed every 1/16 frames. However, the PF is preferable when the RLS is used and can be resumed every $1 / 32$ of the frame size.
\end{abstract}

\section{General Terms}

Data Communication, Equalization and Adaptive Filtering

\section{Keywords}

MIMO-OFDM, Channel Equalization, LMS, VSSLMS, RLS

\section{INTRODUCTION}

The increasing demand for high data rate requires a bandwidth that should be higher than coherence bandwidth. This case becomes worse in modern wireless communication that becomes the most widely used areas of communication. The wireless communication channel usually suffers from fading that cannot be flat fading because the shortage of bandwidth. One promising solution to such problem is the OFDM technology that can be used to make communication channel fading flat. With the OFDM system, the main high data rate stream is divided in many lower data rate sub streams. Each sub stream sent on sub channel where the main system or channel bandwidth is divided. These sub channels should be orthogonal to each other to avoid the problem of inter-symbol interference (ISI) [1]. Further enhancements to the OFDM system can be achieved by combining the MIMO technique with the OFDM system [2,3]. However, even with the use of MIMO-OFDM digital transmission system, there is a need for channel equalization to reduce the effects of ISI and enhance further the system performance $[4,5,6]$.

The ISI arises because of limited band channel and because of the effect of amplitude and phase distortion. Furthermore, the channel distortion is time variant that makes the mobile fading channel. Therefore, the adopted equalizers should be adaptive that have the capability to track the time varying characteristics of the mobile channel [7]. These adaptive require a taps weight adjustment process to achieve the channel equalization. This process of adjustment requires a training sequence mode to adjust equalizer coefficients in accordance with the adaptive filtering algorithm that attempt to reach certain criterion such as mean square error between the received signal and a desired signal that should be known in the training mode [4]. In the training mode, there are two methods to transmit a training sequence (Known by the receiver), the Full Frame (FF) method uses one frame from sets of frames as desired signal and the Part Frame (PF) method uses part of frame as desired signal.

\section{LINEAR ADAPTIVE EQUALIZER}

An equalizer is usually implemented and simulated at the baseband or at IF stage in a receiver. The equalizer is implemented in the form of a linear transversal filter. The type of equalizer depended on the adaptive algorithm, which acts the up data weights equation. The most important types of traditional adaptive equalizers are LMS, RLS, Sign LMS and VSSLMS.

\subsection{Least Mean Square (LMS) Algorithm}

The equalizer is assumed to be constructed from $\mathrm{N}$-taps linear transversal filter with weights computed using the LMS algorithm as shown in Fig. 1. The iterative formulas of the steepest descent method based on LMS algorithm can be defined by:

$$
y(n)=W^{T} x(n)=x^{T} W
$$

where, $\mathbf{x}(n)$ is the filter input; $y(n)$ is filter output and $\mathbf{w}$ is filter weights. The filter weights are adjusted using the LMS algorithm given by;

$$
w(n+1)=w(n)+2 \mu e(n) x(n)
$$

where $\mu$ is the step size, $e(n)$ is the error between desired $d(n)$ and the output signal $y(n)$ is computed using;

$$
e(n)=d(n)-y(n)
$$

It is well known that the LMS algorithm executes quickly, but converges slowly. Its implementation requires, $2 \mathrm{~N}+1$ multiplications [8]. 


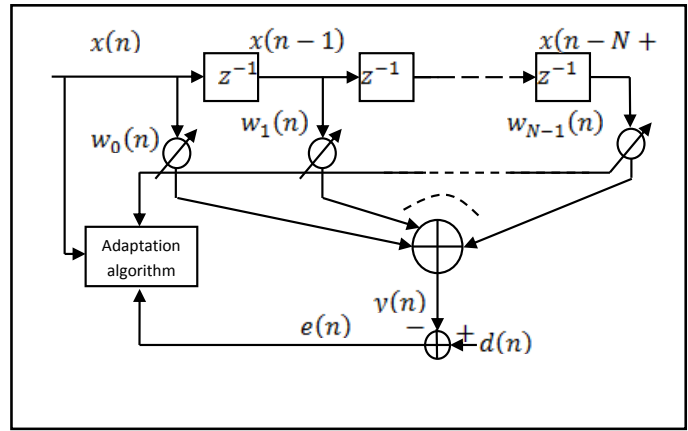

Fig. 1. Adaptive transversal linear filter

\subsection{Variable Step Size Least Mean Square (VSSLMS) Algorithm}

The adoption of variable step size LMS can enhance the convergence speed of the LMS without affecting the convergence precision. Furthermore, variable-step-size LMS algorithms are more robust to variability of the input signal's statistics. In such variable step size LMS algorithm, the initial stages of the training phase is to start with a larger step size in order to have fast convergence speed. However, as the algorithm approaches the steady state where error between the received signal and the desired signal becomes small, smaller step is used. The variable step-size LMS abbreviated as (VSSLMS) algorithm is an effective solution to this problem (Shin and Lee, 1985; Harris, Chabries, and Bishop, 1986). With VSSLMS algorithm, the new tap weight at time instant $\mathrm{t}=\mathrm{nT}$, of the $i$ th element of the tap-weight of the vector $\mathbf{w}(n)$ can be calculated using;

$w_{i}(n+1)=w_{i}(n)+2 \mu_{i}(n) e(n) x(n-i)$

for $\mathrm{i}=0,1 \ldots \mathrm{N}-1$, where $\mathrm{e}(\mathrm{n})$ is the error between desired $d(n)$ and the output signal $y(n), \mu i(n)$ is the a parameter that with the step size at iteration $\mathrm{n}$. The step-size parameter $\mu_{\mathrm{i}}(\mathrm{n})$ is updated upon the variation in e(n). Initially the stochastic gradient term;

$g_{i}(n)=e(n) x(n-i)$

is calculated over the successive iterations of the algorithm. Next, the value of gradient term will decide whether $\mu \mathrm{i}(\mathrm{n})$ is increased or decreased. If the gradient consistently positive, $\mu \mathrm{i}(\mathrm{n})$ is increased and if the gradient in the consistently negative, $\mu \mathrm{i}(\mathrm{n})$ is decreased [8]. This changes of gradient occurred when the convergence is not achieved. However, as the adaptive filter tap weights converge, the averages of the stochastic gradient terms $\mu_{\mathrm{i}}(\mathrm{n})$ approach zero. The decreases in the gradient will result in gradual decrease in the step-size to some minimum values till an optimum value reached otherwise it will result in increase of the corresponding stepsize parameters [8]. The following equation reflects the effect of gradient on the step size parameter.

$\mu_{i}(n)=\mu_{i}(n-1)+\rho g_{i}(n-1)$

where $\rho$ is a small positive step-size parameter.

\subsection{Sign Least Mean Square (SignLMS) Algorithm}

SignLMS algorithm is a modification to the conventional LMS. This modification is achieved by replacing e(n) with its sign in Eq. 3, such as: $w(n+1)=w(n)+2 \mu \operatorname{sign}(e(n)) x(n)$

where $\mu$ is a constant. The replacement of e(n) by its sign will simplify the implementation of the conventional LMS formula since no multiplication would be required in the implementation of SignLMS algorithm [8].

\subsection{Recursive Least Squares (RLS) Algorithm}

In the RLS algorithm the weighting factor $\rho n(k)$ is chosen as:

$$
\rho_{n}(k)=\lambda^{n-k} \text { for } k=1,2, \ldots . ., n
$$

where $\lambda$ is a positive constant called forgetting factor. The value of $\lambda$ is with ordinary LMS is equal to 1 whereas RLS algorithm it should be $\lambda<1$ but close to 1 . The weighting factor that should be chosen less than 1 produces an algorithm that puts more emphasis on the recent samples of the observed data and tends to forget the past. The taps weight of the RLS can be updated using;

$$
\hat{W}=\hat{W}(n-1)+k(n) e_{n-1}^{\wedge}(n)
$$

where $\mathrm{k}(\mathrm{n})$ gain vector and

${\hat{e_{n-1}}}^{\wedge}(n)=d(n)-\hat{\lambda}_{n-1}(n)$

Details about gain vector computation can be found elsewhere [8]. The RLS algorithm converges quickly, but its complexity grows with the square of the number of weights.

\section{EQUALIZERS TRAINING}

Figure 2 presents the details of a baseband transmission system, equipped with an adaptive equalizer. Usually, with equalizer training, the receiver should know the transmitted data symbols and for this reason it has been mentioned as the desired signal samples that is essential for the equalizer taps weight adjustment. During the training mode or initialization period, the transmitter sends a sequence of training symbols that are known to the receiver [8].

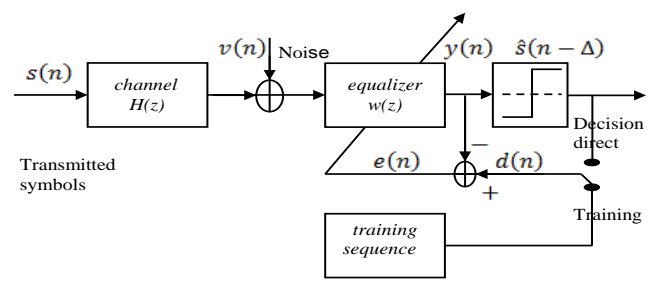

Fig 2: Baseband data transmission system equipped with an adaptive channel equalizer

There are two modes of operation for adaptive equalizer, namely, the training mode and Decision-Directed mode (DDM). The equalizer is adjusted under the training mode where the training sequence transmitted through the channel. A synchronized version of this training sequence is generated at the receiver, it is applied to the equalizer as the desired response, when the training process is completed, and the adaptive equalizer is ready for normal data transmission [9]. In the training mode, there are two methods to transmit training sequence, the Full Frame (FF) method uses one frame from sets of frames as desired signal and the Part Frame (PF) method uses part of frame as desired signal. According to the influence of the surroundings environment, different percentage of training sequence can be used. The ratio of 
training sequence that are used in this paper are: 1/8, 1/16 and $1 / 32$. Figure 3 shows the two methods of training mode with ratio $1 / 8$

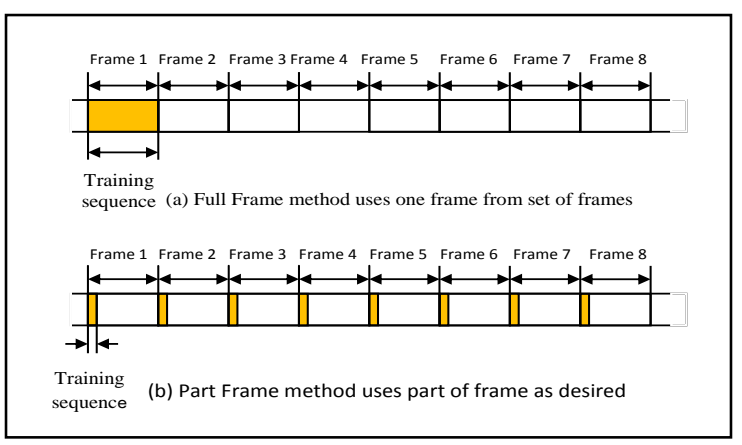

Fig 3: Two methods of training mode with ratio 1/8

\section{SYSTEM IMPLEMENTATION AND EVALUATION}

The MIMO-OFDM system was implemented with the aid of MATLAB/SIMULINK program shown in Figure 4 with parameters of each component as shown in Table 1. In this implementation, a binary data passe through convolutional encoder, 16-QAM modulation, Alamouti encoder $(2 \times 2)$ and OFDM modulation. The parameters which be applied to OFDM modulation are according to the wireless standard IEEE 802.16 and then the modulated signal is passed through a multipath Rayleigh fading channel with Doppler frequency equal to $(100 \mathrm{~Hz})$ and subjected to AWGN. At the receiver side, OFDM demodulation, Alamouti combiner $(2 \times 2)$, 16QAM demodulation are performed on the received signal. Next, an adaptive equalizer with the number of taps equal to (4) and Viterbi decoder are used to recover the transmitted signal.

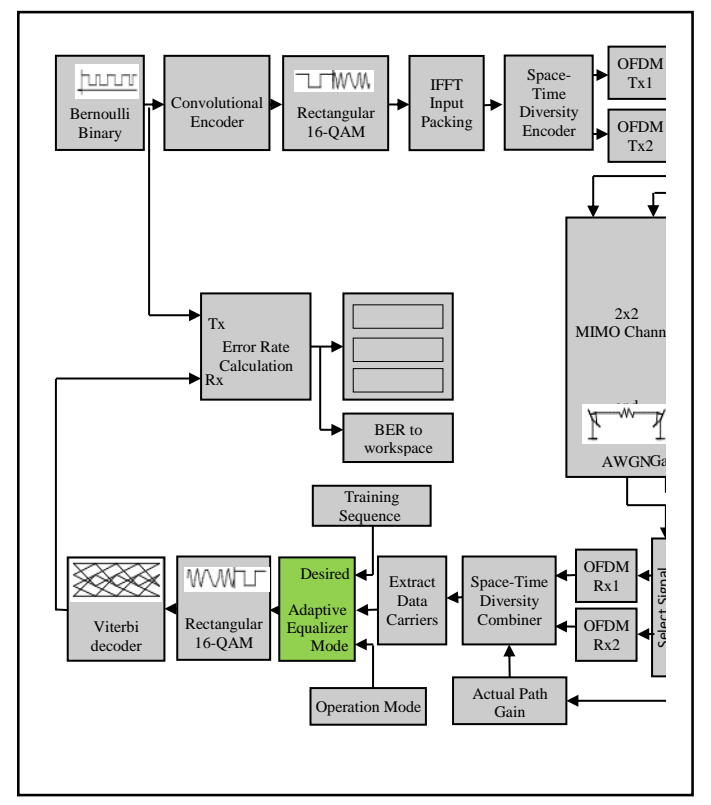

Fig 4: The MIMO-OFDM system with an equalizer

By using Monte Carlo tool with the model described in Figure 4 , the performance of two methods of training mode with different training ratio are achieved. The Bit Error Rate (BER) is taken at $\left(10^{-5}\right)$ as reference value to show the advantages gained in EbNo by each type of equalizer and training modes.
Table 1. Simulation parameters specification

\begin{tabular}{|l|l|}
\hline Parameter & Value \\
\hline Data Source (Sample time) & $1.25 \times 10^{-8} \mathrm{sec}$ \\
\hline coding & $1 / 2$ Convolution Encoder \\
\hline Data rate & 50 Mbps \\
\hline Modulation/ Demodulation: & Rectangular 16-QAM \\
\hline FFT size: & 256 symbol \\
\hline Cyclic prefix of OFDM symbol: & $1 / 8=32$ symbol \\
\hline Type of MIMO: & OSTBC $2 \times 2$ conf. \\
\hline Doppler spectrum type: & Jakes \\
\hline Adaptive Equalizers types: & $\begin{array}{l}\text { LMS, VSSLMS, SignLMS } \\
\text { and RLS }\end{array}$ \\
\end{tabular}

For LMS equalizer, it has been found that the optimum step used for best performance is equal to (0.002). Test with such step size shown Figure 5 suggest that at $\left(\mathrm{BER}=10^{-5}\right)$, the Full Frame method and training ratio (1/8) is better than Part Frame method at the same training ratio. Furthermore, the EbNo equal to $(9.325 \mathrm{~dB})$ in FF method while in PF method equal to $(9.715 \mathrm{~dB})$. Extra test with training ratio $(1 / 16)$ shows that also the Full Frame method is better than Part Frame and the $E b N o$ equal to $(9.49 \mathrm{~dB})$ in $\mathrm{FF}$ method while in $\mathrm{PF}$, the equalizer fails to converge.

Tests of VSSLMS equalizer, show that minimum and maximum step size are 0.006 and 0.005 successively and the increment step size is 0.0001 give the best performance. It has been shown from Figure 6 that at $\left(\mathrm{BER}=10^{-5}\right)$, the Full Frame method with training ratio of (1/8) is better than Part Frame method with the same training ratio. Furthermore, the EbNo equal to $(9.217 \mathrm{~dB})$ in FF method while in PF method, it is equal to $(9.955 \mathrm{~dB})$. Test with a training ratio of $(1 / 16)$, the Full Frame method is better than Part Frame too and the achieved $E b N o$ is equal to $(9.245 \mathrm{~dB})$ in $\mathrm{FF}$ method while in $\mathrm{PF}$ the $E b N o$ it is equal to $(9.956 \mathrm{~dB})$. Test with training ratio of (1/32) shows also that the Full Frame method is better than Part Frame and that achieved EbNo is equal to $(9.45 \mathrm{~dB})$ in FF method while in PF, the equalizer fails to converge. Tests with SignLMS equalizer, show that the optimum step size that can be used to give the best performance is equal to (0.004). Furthermore, Figure 7 shows that Full Frame method with training ratio $(1 / 8)$ is better than Part Frame method with the same training ratio. The results also show that the EbNo equal to $(9.51 \mathrm{~dB})$ in $\mathrm{FF}$ method while in $\mathrm{PF}$ method is equal to (9.995 dB). However, the Full Frame method with training ratio $(1 / 16)$ is better than Part Frame. It has been found that the EbNo is equal to $(9.6 \mathrm{~dB})$ in FF method while in PF, the equalizer fails to converge.

Finally, test of RLS equalizer shows that the optimum value for Forgetting Factor that can be used to give the best performance is equal to $\left(0.97\right.$. Figure 8 shows at $\left(B E R=10^{-5}\right)$, the Full Frame method with training ratio of $(1 / 8)$ is better than Part Frame method with the same training ratio. Furthermore, the result shows that the EbNo is equal to (9.269 $\mathrm{dB})$ in FF method while in PF method the EbNo is equal to $(10.08 \mathrm{~dB})$. However, for training ratio of (1/16), the Full 
Frame method is better than Part Frame. The results also show that the $E b N o$ is equal to $(9.6 \mathrm{~dB})$ in the FF method while the $E b N o$ is equal to $(10.427 \mathrm{~dB})$ in PF. Extra test with a training ratio (1/32) shows that the Full Frame method is also better than Part Frame. The test shows that the EbNo is equal to (9.8 $\mathrm{dB})$ in $\mathrm{FF}$ method while the EbNo equal to $(11.8 \mathrm{~dB})$ in the PF.

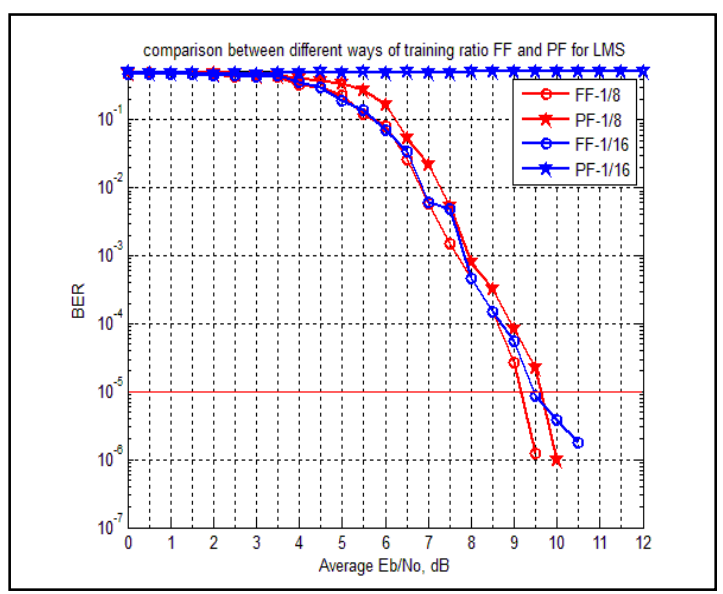

Fig 5: Comparison between Full and Part Frame for LMS equalizer

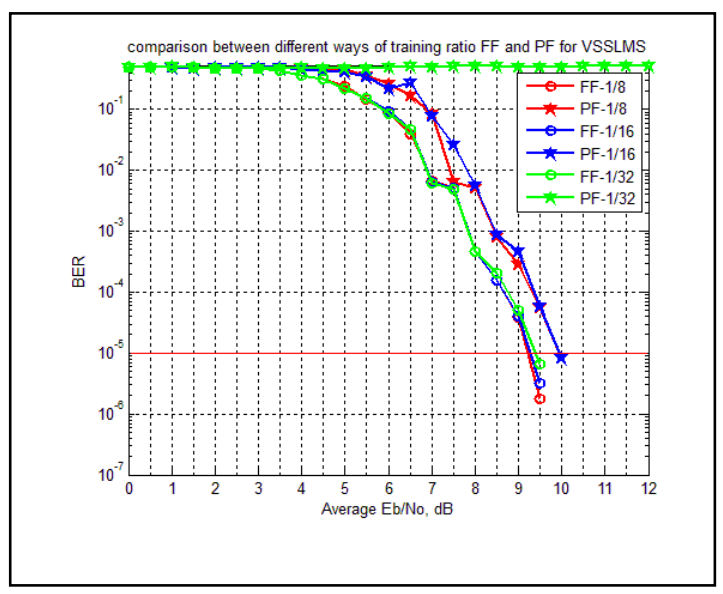

Fig 6: Comparison between Full and Part Frame with VSSLMS

equalizer

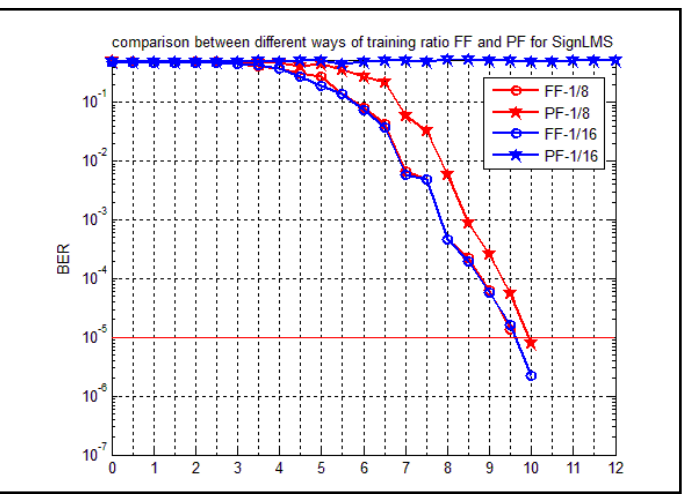

Fig 7: Comparison between Full and Part Frame for SignLMS equalizer

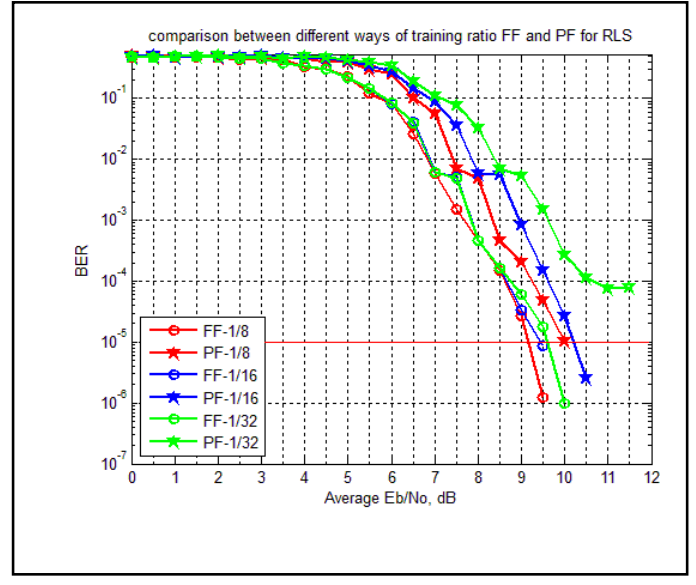

Fig 8: Comparison between Full and Part Frame for RLS equalizer

Finally, a summary that shows a clear comparison between LMS, VSSLMS, SignLMS and RLS adaptive equalizers with FF and PF training modes is shown in Table 2. The table shows the required $E b N o$ for each type of equalizer to achieve the $\mathrm{BER}=10^{-5}$ that reflect the advantages gained by each type over the other. This result is also based on the performance comparison between all types of equalizers and the case of no equalizer used as shown in Figure 9. It is clear that VSSLMS is the preferable type of adaptive equalizer because of the least EbNo required. The results also indicate that $\mathrm{FF}$ training mode is better than that of the PF training mode in EbNo required together with VSSLMS the training can be resumed every $1 / 32$. The slight gain achieved by the VSSLMS in EbNo together with the fair comparison between the four types of equalizers suggests an extra comparison between the equalizers in terms of convergence speed and complexity as shown in Table 3. The results presented in Table 3 show that VSSLMS has the advantages of convergence speed together with the less consuming energy. However, the signLMS and LMS have less complexity, but very low and low convergence speed.

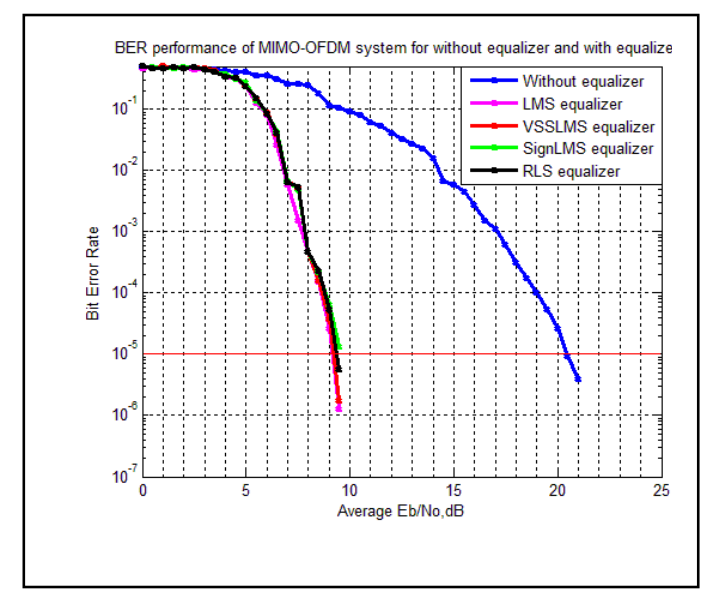

Fig 9: BER performance of MIMO-OFDM system 
Table 2. Comparison of values of EbNo (dB) for different equalization techniques and training modes

\begin{tabular}{|l|l|l|l|l|}
\hline $\begin{array}{c}\text { Training ratio } \\
\text { sequence }\end{array}$ & LMS & VSSLMS & SignLMS & RLS \\
\hline 1/8 Full Frame (FF) & 9.325 & 9.217 & 9.51 & 9.269 \\
\hline 1/8 Part Frame (PF) & 9.715 & 9.955 & 9.995 & 10.08 \\
\hline $1 / 16$ Full Frame (FF) & 9.49 & 9.245 & 9.6 & 9.6 \\
\hline $1 / 16$ Part Frame (PF) & Fail & 9.956 & Fail & 10.427 \\
\hline $1 / 32$ Full Frame (FF) & Fail & 9.45 & Fail & 9.8 \\
\hline $1 / 32$ Part Frame (PF) & Fail & Fail & Fail & 11.8 \\
\hline
\end{tabular}

Table 3. Comparison between the four algorithms

\begin{tabular}{|l|c|c|c|}
\hline Algorithm & $\begin{array}{c}\text { Least } \\
\text { consuming } \\
\text { (EbNo)dB }\end{array}$ & $\begin{array}{c}\text { Convergence } \\
\text { speed }\end{array}$ & $\begin{array}{c}\text { No. of } \\
\text { multiply } \\
\text { operations [8] }\end{array}$ \\
\hline LMS & 9.325 & Low & $2 \mathrm{~N}+1$ \\
\hline VSSLMS & 9.217 & High & $4 \mathrm{~N}+1$ \\
\hline SignLMS & 9.51 & Very Low & No need \\
\hline RLS & 9.269 & High & $4 \mathrm{~N}^{2}$ \\
\hline
\end{tabular}

\section{CONCLUSIONS}

The paper investigated the effect of using four types of adaptive equalizers, LMS, VSSLMS, SignLMS and RLS adaptive equalizers with two different modes of training, full frame and part of frame mode. These types of equalizers have been investigated under multipath Rayleigh fading channel, operating with MIMO-OFDM system. The results of computer simulation investigate the equalizers dependence on how much EbNo required to give $\left(\mathrm{BER}=10^{-5}\right)$, equalizers weights convergence speed and the complexity. The results show that the Full Frame method training mode is better than Part Frame method for all types of equalizers. Furthermore, the results also show that VSSLMS algorithm is the best when it uses Full Frame method because it consumes least EbNo and has fast weights convergence speed compared to other types of equalizers. However, Part Frame method with RLS algorithm is best because it continues to work until the ratio of $1 / 32$ while the rest algorithms fail at the same ratio.

It can be concluded from the results that the choice of equalizer training mode is essential and depend on the complexity and speed of convergence. When complexity is not an issue to be considered, the RLS is the best choice whereas when the complexity is the issue to be considered the VSSLMS will be the choice.

\section{REFERENCES}

[1] Popovski, P. et al 2013, Novel radio link concepts and state of the art analysis, Petra Weitkemper (DOCOMO Euro-Labs).

[2] Saleem, S. 2012 Optimization of Channel Estimation Algorithms for MIMO-OFDM based LTE-Advanced, IJCSI, Volume 2.

[3] Kansal, B. L. 2012 Performance Analysis of MIMOOFDM by Spatial Diversity with STBC4, International Journal of Computer Applications Volume 48- No.20, 2012.

[4] Malik, G. and Sappal, A. S. 2011 Adaptive Equalization Algorithms:An Overview. (IJACSA) Vol. 2, No.3.

[5] Mahmood, L., Shirazi, S. F., Naz, S., Shirazi, S. H., Razzak, M. I., Umar, A I., and Ashra, S. S. 2015 Adaptive Filtering Algorithms for Channel Equalization in Wireless Communication, Indian Journal of Science and Technology, Vol 8(17).

[6] Ahmed, M. A, Jimaa S. A., and Abualhaol, I. Y. 2012 Performance Enhancements of MIMO-OFDM System Using Various Adaptive Receiver Structures, International Journal of Computer and Information Technology (2277 - 0764) Volume 01- Issue 01.

[7] Jagan V.; Murali K.; Krishna K. Rajeswari, R. 2011 Performance Analysis of Equalization Techniques for MIMO Systems in Wireless Communication, International Journal of Smart Home Vol.4, No.4.

[8] Farhang-Boroujeny B. 2013 Adaptive filters: theory and applications, 2nd Edition, Wiley.

[9] Kim J. S et al. 2015 An Adaptive Equalizer for HighSpeed Receiver using a CDR-Assisted All-Digital Jitter Measurement", Journal of Semiconductor Technology and Science, Vol.15, No.2. 